\title{
Model Identity Card (MIC) for Simulation Models
}

\author{
Saïna Herssand, Eric Landel, Jean-Marc Gilles and Joe Matta
}

\begin{abstract}
Modeling a complex system implies the integration of different simulation models in various fields of expertise. These models should communicate with each other to simulate the behavior of the whole system. In this multidisciplinary context, the actors involved in the modeling process should deal with three main problems. Firstly, in order to reduce ambiguity, they need a common vocabulary and format to describe their models in a less informal way. Secondly, in order to reduce the cost of lately correction, any potential incompleteness and inconsistency problems related to the models should be identified in the early phases of creation and integration of models. Thirdly, the characterization of simulation models should allow actors to reuse existing models more efficiently. In this poster, we propose a common framework called Model Identity Card (MIC) to specify and characterize simulation models contents and interfaces. This new concept is implemented in arKItect (a MBSE tool) to facilitate the knowledge sharing between different actors. It allows users to reduce time to get a correct model by checking the completeness and consistency of their models throughout the modeling process. An industrial test-study in automotive industry is presented to illustrate the interest of the proposed approach.
\end{abstract}

Supporting multidisciplinary vehicle modeling by Göknur Sirin supervised by Bernard Yannou -Châtenay-Malabry, École Centrale de Paris and Eric Landel, Renault.

S. Herssand $(\square) \cdot$ J. Matta

Knowledge Inside, 7B rue Jean Mermoz, 78600 Versailles, France

e-mail: saina.herssand@k-inside.com

J. Matta

e-mail: joe.matta@k-inside.com

E. Landel · J.-M. Gilles

Renault Group, 1 avenue du Golf, 78280 Guyancourt, France

e-mail: eric.landel@ renault.com

J.-M. Gilles

e-mail: jean-marc.gilles@ renault.com 